\title{
Transmission Electron Microscopy Studies of Carbonaceous Chondrites which Experienced Experimentally Simulated Space Weathering Effects
}

\author{
M. S. Thompson ${ }^{1}$, L. P. Keller ${ }^{1}$, R. Christoffersen ${ }^{2}$, M. J. Loeffler ${ }^{3}$, R. V. Morris ${ }^{1}$, T. G. Graff ${ }^{2}$, and Z. \\ Rahman $^{2}$. \\ 1. ARES, NASA Johnson Space Center, Houston, TX, USA. \\ 2. Jacobs, NASA Johnson Space Center, Mail Code XI3, Houston, TX, USA. \\ 3. NASA Goddard Space Flight Center, Greenbelt, MD, USA.
}

Introduction: Mineral grains on the surfaces of airless bodies such as the Moon and asteroids are continually modified due to their exposure to interplanetary space. Radiation processing via solar energetic ions from the solar wind and micrometeorite impacts collectively alter the microstructure, chemical composition, and optical properties of soils grains on airless planetary surfaces [1]. This phenomenon is known as space weathering, and understanding the nanoscale effects that result from this processing is essential for interpreting spectroscopic data collected by remote sensing spacecraft.

Space weathering features have been studied in samples returned from the lunar surface via the Apollo missions, and more recently from the surface of near-Earth asteroid Itokawa, returned by the Hayabusa mission, e.g., [2,3]. In addition to analyzing returned samples, space weathering processes have been simulated in the laboratory using experimental techniques. Pulsed laser irradiation is used to simulate the energy transfer associated with a micrometeorite impact, e.g., [4]. In both returned and experimental samples the microstructural and chemical effects are evident on the nano- and microscale, including: partial and complete amorphization, vesiculated textures, chemically distinct deposits on grain surfaces, and nanophase Fe particles in grain rims and interiors. While work has been done to understand the effects of space weathering on the Moon and Itokawa, studies targeting another type of parent body are noticeably deficient: carbonaceous asteroids. Understanding the effect of space weathering processes on both the organic and inorganic components of carbonaceous materials will be critical for understanding the nature of samples returned by up-coming missions targeting primitive, organic-rich bodies (e.g., OSIRIS-REx and Hayabusa2). Here we present nanoscale analyses of the effects of simulated micrometeorite impacts on carbonaceous materials relevant for these upcoming sample return missions.

Sample and Methods: We performed our experiments using the CM2 Murchison meteorite, which has been identified as an appropriate spectral analog for the materials targeted by the OSIRIS-REx and Hayabusa2 missions. We subjected a chip of Murchison to pulsed laser irradiation to simulate a micrometeorite impact, rastering the laser once over the surface of the sample (and area of several $4 \mathrm{~mm}^{2}$ ). We placed a glass slide above the sample to enable the collection of the resultant recondensed vapor plume. A JEOL 7600F field emission scanning electron microscope (SEM) with x-ray detector system was used to study the morphological and chemical effects of laser irradiation on the meteorite chip. We used the focused ion beam (FIB) to extract electron transparent sections from both the irradiated sample and the vapor deposit for analysis in the transmission electron microscope. We used the FEI Quanta 3D FIB to extract sections which were analyzed using the JEOL 2500SE scanning transmission electron microscope (STEM), equipped with bright-filed (BF) and dark-field) STEM detectors and a Thermo thin window energy-dispersive X-ray (EDX) spectrometer. Quantitative spectrum imaging was completed using a $2 \mathrm{~nm}$ probe. 
Results and Discussion: SEM analysis of the meteorite matrix after experiencing laser irradiation shows textures consistent with melting and possible outgassing of volatiles (e.g., burst vesicles). TEM analysis of the extracted FIB section show an amorphous melt layer on the surface of the underlying matrix material, with individual melt blebs up to $500 \mathrm{~nm}$ in thickness. This melt layer exhibits a vesiculated texture and EDX mapping indicate the melt layer is composed of an Fe-Mg-silicate, with localized concentrations of S. The melt blebs contain nanophase Fe and Fe-Ni-Sulfide particles (Fig. 1) measuring up to $200 \mathrm{~nm}$ in size. HRTEM imaging show lattice fringes with d-spacings of $5.8 \AA$, consistent with pentlandite $\left((\mathrm{FeNi})_{9} \mathrm{~S}_{8}\right)$ (Fig. 1). Phyllosilicate phases exhibit localized amorphization within the outermost $800 \mathrm{~nm}$ of the surface of the section, but maintain crystallinity at depth (Fig. 1). TEM analysis of the FIB section extracted from the vapor deposit reveals the presence of a chemically stratified deposit of non-uniform thickness (30-350 nm), indicative of multiple distinct emplacement events (Fig. 2). EDX maps show thin, uniform, volatile-rich deposits (e.g., Fe and S) and thicker, pancake-shaped, more refractory $(\mathrm{Mg}, \mathrm{Si}, \mathrm{Fe}$ and occasionally $\mathrm{Ca}$ ) features. These deposits are likely representative of recondensation of the initial vapor cloud and melt droplets produced by the laser irradiation impacting the glass slide, respectively. There are vesicles and nanoparticles present throughout both the melt and vapor deposits. Selected area electron diffraction patterns collected for nanoparticle-bearing deposits indicate at least three phases are present: troilite (FeS), pentlandite, and magnetite $\left(\mathrm{Fe}_{3} \mathrm{O}_{4}\right)$. The results presented here indicate that micrometeorite impact processes produce textures consistent with melting of the substrate, and generate a chemically distinct vapor plume which subsequently recondenses. The high water and volatile content of carbonaceous materials produce vesiculated textures and the identification of nanophase Fe-Sulfides is consistent with previous experiments suggesting the importance of these phases in asteroidal space weathering.

\section{References:}

[1] B Hapke, Journal of Geophysical Research-Planets 106 (2001), p. 10,039 .

[2] L. P. Keller and D. S. McKay, Geochimica et Cosmochimica Acta 61 (1997) p. 2331.

[3] T. Noguchi et al, Meteoritics and Planetary Science 49 (2014) p. 188.

[4] Sasaki, S. et al, Nature 410 (2001) p. 555.
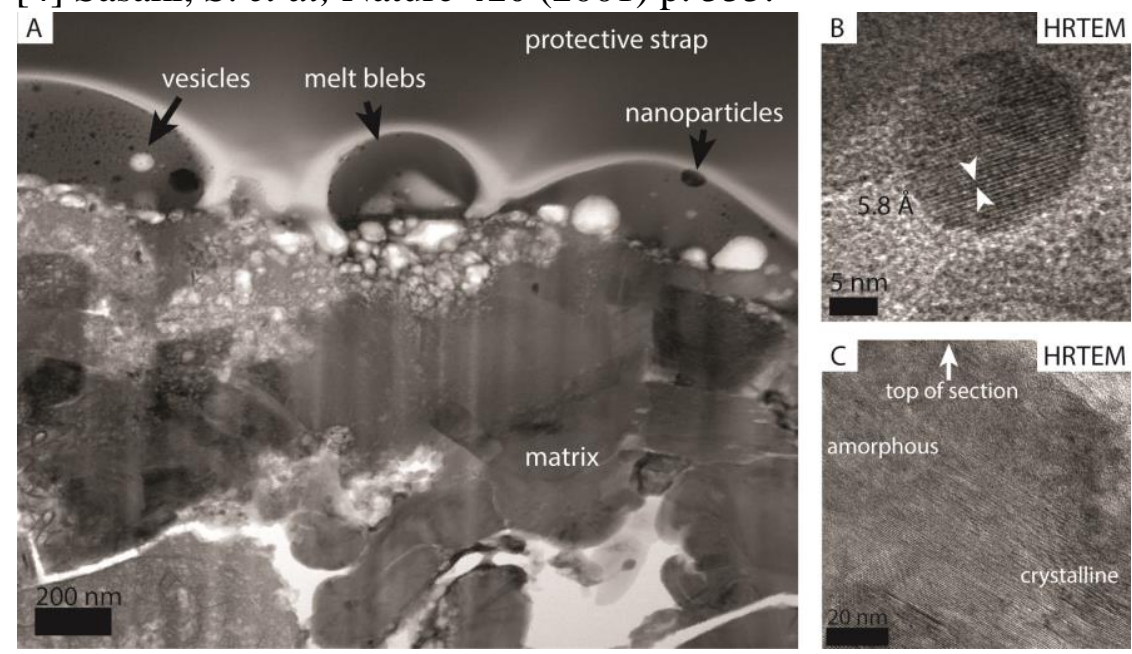

Figure 1: A) BF STEM image showing melt blebs, vesicles and nanoparticles. B) HRTEM image showing lattice fringes indicating pentlandite. C) HRTEM image showing localized amorphization.

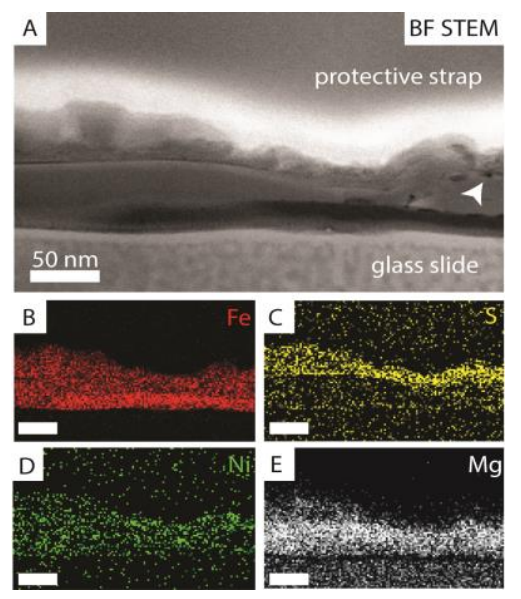

Figure 2: A) BF STEM image showing the complex stratigraphy of the melt and vapor deposit. The white arrow indicates the location of a nanophase Fe-Ni-S grain. EDS maps of the region showing the distribution of $\mathrm{B}$ ) $\mathrm{Fe}, \mathrm{C}) \mathrm{S}$, D) $\mathrm{Ni}$, and E) Mg. $50 \mathrm{~nm}$ scale bars. 